\title{
Augmented reality as a design tool for mobile interfaces
}

\author{
Olav W. Bertelsen \& Christina Nielsen \\ University of Aarhus \\ Department of Computer Science \\ Aabogade 34, DK-8200 Arhus N. Denmark \\ +4589423188 \\ \{olavb|sorsha\}@daimi.au.dk
}

\begin{abstract}
This paper challenges user interface paradigms for mobile devices, by using the technical classification of augmented reality interfaces as a 'thinking tool' to develop ideas for interaction with mobile devices. The paper presents future work scenarios from a wastewater treatment plant embodying PDA applications derived from the classification of augmented reality interfaces. The focus on physical interaction with objects of work and with the mobile device provides us with a range of interaction styles, based on e.g. gestures and manipulation of objects. Furthermore, issues of transparency and directness are addressed. The future scenarios indicate that the concepts of augmented reality support solving context problems in mobile design.
\end{abstract}

\section{Keywords}

Thinking tools, augmented reality, mobile computing, process control.

\section{INTRODUCTION}

It is widely acknowledged in the field of mobile computing that there is a need for new interaction paradigms $[5,7,12$, 9]. Solving this problem requires insights from a broad range of disciplines, including distributed systems, HCI, CSCW and participatory design [5]. Established concepts in desktop computing are not scaleable. E.g., the concept of direct manipulation that historically has served as the main vehicle for understanding the graphical workstation is not applicable in the design of small mobile devices [9]. This difficulty is due to the tunnel vision imposed by, otherwise valuable, established cultural norms like the desktop metaphor, the workstation concept, and the general thinking in terms of database access.

Augmented reality is an approach to information systems design augmenting physical objects instead of replacing or representing them by purely computer based systems. The argument is that non-computer based artefacts in the workplace often mediate work in subtle ways that are impossible to transfer to new computer based artefacts [10].

\section{To Appear in Proceedings of the ACM DIS '00 Conference,} Aug. 17-19, 2000, Brooklyn, New York
In a work-oriented terminology [4, 14], augmentation can be understood as a basic feature of human action in the sense that human action always is mediated by historically developed artefacts which are invisible during use. Augmentation and augmented reality can be seen as a general attitude to design, realised through refinement of mediating artefacts, acknowledging the historical crystallisation of work into successive generations of artefacts [1,2].

Mackay [10] introduces augmented reality as a classification of three technical approaches to design of interactive devices, spanning a continuum of technical substrates for mixed environments: augmenting the user, the physical object and the environment (see Table 1 below). These strategies describe the technical locus of the interface assuming the analytical separation of function and interaction in the computer artefact. From the work-oriented point of view, introducing a new artefact is always a matter of augmenting the acting subject.

In this paper, we explore new interface principles for small mobile devices derived from the concept of augmented reality interfaces. We use the concept as a tool for divergent thinking, in line with the use of metaphors in participatory design [11], and springboards in developmental work research [6]. By definition, the concept of augmented reality cannot be applied to small mobile devices; thus we abstract defining features from the three directions in augmented reality interfaces and apply them in the different technical setting. Subsequently, the principles are further investigated through future scenarios of PDA support for wastewater treatment work built on the augmented reality classification transformed to small mobile interfaces.

\section{AUGMENTED REALITY IN A PDA}

In applying the concept of augmented reality as a thinking tool for the design of mobile artefacts, the specific technical substrates in which augmented reality interfaces are implemented are bracketed out. What we hold on to is the principle of interaction with or through physical objects (already existing) in the work setting. Below, we explore the three directions in augmented reality interfaces described by Mackay [10]. We use this classification to develop metaphors for mobile applications not based on the idea that the PDA is miniature workstation. 


\begin{tabular}{|l|l|l|l|}
\hline Augment & Approach & Technology & Application \\
\hline User & Wear devices on the body & $\begin{array}{l}\text { VR helmets Goggles Data } \\
\text { gloves }\end{array}$ & $\begin{array}{l}\text { Medicine, } \\
\text { Field service, Presentations }\end{array}$ \\
\hline Physical Object & Embed devices in objects & $\begin{array}{l}\text { Intelligent bricks, Sensors, } \\
\text { receptors, GPS, electronic } \\
\text { paper }\end{array}$ & $\begin{array}{l}\text { Education, } \\
\text { office facilities, Positioning }\end{array}$ \\
\hline $\begin{array}{l}\text { Environment surrounding } \\
\text { objects and users }\end{array}$ & $\begin{array}{l}\text { Project images and record } \\
\text { remotely }\end{array}$ & $\begin{array}{l}\text { Video cameras, Scanners, } \\
\text { Graphics tablets, Bar code } \\
\text { readers, Video projectors. }\end{array}$ & $\begin{array}{l}\text { Office work, Film-making, } \\
\text { Construction, Architecture }\end{array}$ \\
\hline
\end{tabular}

Table 1. Examples of augmented reality interfaces, with relevant technologies and applications (adopted from Mackay 1998).

\section{Augmenting the user}

The strategy of augmenting the user means that the interface to the computer system is attached to the user as e.g. VR helmets, goggles or data gloves. When implemented in a PDA, this strategy is transformed to applications where the PDA is physically attached to the user (possibly hand held). The PDA functions as "glasses" extending the users perceptual capabilities in (into) the domain (work object), and as "gloves" extending the users capabilities in acting onto the domain. Interaction may be carried out through gestures performed holding the PDA.

Because the PDA acts as an extension to the user, the connection between PDA and object is hidden and indirect. Within this strategy, we may include context specific access to a more general information system.

The augmenting the user approach to PDA design could lead to applications resembling a well-designed remote control for a TV set. The remote control is in the TV viewing situation a transparent, almost invisible, extension of the viewer. The viewer manipulates the TV-set directly through gestures with the remote control. This sense of direct manipulation is obtained through the layout and shapes of the buttons.

Another existing example is the way wireless controls of lifts are attached to the worker operating the machine from a distance.

\section{Augmenting the object}

The strategy of augmenting the object means that the interface to the computer system is attached to, or embedded in the object. When implemented in a PDA, this strategy is transformed to applications where the PDA is provisionally attached to the object. Interaction with the PDA (or the wider information system) is carried out through manipulation of the physical object to which the PDA is attached, and through showing information on the object by way of the PDA display. Thus, the PDA augmenting the object, turns the physical object into an interaction device and at the same time makes the object more accessible for the user.
Because the PDA becomes embedded in, or rather attached to, the object, the connection between the PDA and the object is visible and direct.

The augmenting the object approach to PDA design could lead to applications resembling the help and guidance system in many modern photocopiers. When a compartment is open, the display on the copier indicates which one and points to its physical location. In case of a paper jam, the computer system guides the user through a sequence of physical actions triggered by the user manipulating e.g. hatches in different areas of the machine.

Another approach based on augmenting the object could be to take the idea of augmented paper [10] to the extreme by simply substituting existing notepads with a PDA based application. Obviously, this is not augmented reality in any strict sense of the concept.

\section{Augmenting the environment}

The strategy of augmenting the environment means that the interface to the computer system is embedded in the environment surrounding users and objects, projecting images and recording remotely. When implemented in a PDA, this strategy is transformed to applications where the PDA is provisionally placed in the environment of the work situation. Interaction with the PDA (or the wider information system) is carried out through manipulation of the physical objects that are tracked, by means of technologies in a continuum from bar code readers to cameras with image recognition build into the PDA, and through projecting information onto the object. For the latter, a lot of development of basic technology is required - although more realistic, low cost solutions are being developed, e.g. the Gesture Laser [15].

Because both user action and objects have to be tracked, and images ideally should be projected onto objects, this strategy is the most demanding to implement.

The augmenting the environment approach to PDA design could lead to applications resembling the set-up at a workstation at an assembly line, where mechanical/electronic devices are assembled. Such devices 
typically consist of a number of printed circuits, connectors and shielding. The assembly is done by hand and requires different electronic circuits depending on the type of device. At a specific workstation for assembling flow meters we have seen that, to aid the assembly procedure, each primary component (not including screws, bolts and nuts) is equipped with a bar code, which must be entered into the computer responsible for testing the assembled product. The assembly procedure cannot be completed until all primary components have been entered into the system, and the computer clearly indicates if wrong components have been scanned. In a sense, the computer is tracking the physical objects required for a specific flow meter and even though, it has no direct, online connection to the components, e.g. through sensors.

\section{EXPLORING THE THREE STRATEGIES}

During the past year, we have been engaged in a study of wastewater treatment plants. Wastewater treatment is a low risk process running continuously day and night. Work in the plant is distributed to a degree where it seems to go on elsewhere, no matter where you look. People working on the plant have two general modes of relating to the plant and the process. Totally mediated, i.e. through the process control system or totally unmediated, by looking at and smelling the water. The workers do not seem to have one favourite tool; e.g. illustrated by one of the workers making a sludge sample in a used plastic glove and stirring the sample with a pen. For a more detailed description of the project and the study, see (Bertelsen \& Nielsen 1999).

In order to develop further the interface principles derived from the concepts and classification of augmented reality, we develop scenarios for support of situations in the wastewater treatment plant. The scenarios are based on applications for a standard PDA, in this case a 3Com Palm Pilot $^{\mathrm{TM}}$ IIIx.

Additional features to be built into the PDA are held to a minimum and are as far as possible taken to be the same across the future scenarios. That is to emphasise our focus, namely the new interaction principles for the PDA rather than futuristic gadgetry. Thus the way the PDA is interfacing with the environment is limited to the standard infra red port, a bar code reader, a standard cell phone and a modem.

\section{Augmenting the user}

In implementing the principle of augmenting the user in a PDA supporting work in a wastewater treatment plant, we take the simple approach of the remote control. We use one of the more peculiar situations we observed in our field study, a repair situation, as our example/template to illustrate and develop the approach.

The situation was a case of simple repair work. A wire operating the scrapers in one of the clarifier tanks was broken, causing the tank to shut down automatically. A team of blacksmiths' was called in to fix the problem. As part of the repair, it was necessary to operate the winch. The winch motor was, however, only controllable from a terminal in the central control room. Thus, the smith had to make a telephone call to the central control room which, incidentally, was occupied by a worker, and ask him to start the motor. Unfortunately, this had no effect on the motor. The worker in the control room suspected a failure in the process control system and called the foreman, who was working outside the plant that day, to let him know about the problem with the system. The foreman explained that the winch usually gets stuck if it gets into one of the extreme positions. He asked the worker to tell the smith to call him on the telephone so that he could guide him through releasing the wire. The worker in the control room was now waiting for the smith to call and tell him to start the motor again, but nothing happened until the smith called to inform him that he was done with the repair. In addition to the information on how to unblock the wire, the foreman had explained to the smith how to short circuit a few cables in the motor to bypass the central control of the motor locally.

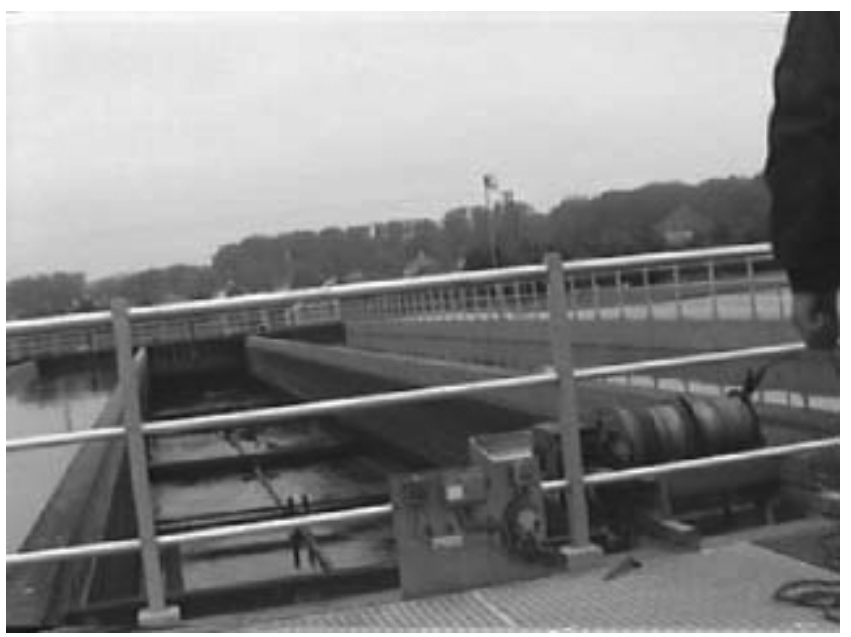

Figure 1: Blacksmith fixing a broken wire in a clarifier tank

By centralising and co-ordinating control of individual component, the process control system ensures that interdependencies are not violated. Mobile control is a highly problematic possibility to integrate into a process control system in that it may violate the purpose of having a process control. However, the situation with the broken wire illustrates the dilemma inherent with centralised control. Either the smith would be on the telephone with somebody in the control room (which is what happens over and over again in all the wastewater treatment plants our collages and we have studied), or he would "hack" the control system, which would be a potential security problem. The challenge is to find a solution that maintain the consistency and security of local control, without 
imposing cumbersome procedures that invites the operators to bypass parts of the system.

This challenge is addressed by the following future scenario with a PDA-application based on the augmenting the user strategy. The "standard PDA" is extended with a bar code reader and a modem for the cellular telephone the smith is carrying anyway. During repair of the wire, when the smith needs to operate the winch he reads the bar code on the winch and calls up the process control system through the telephone. Together with the identification of the particular component, the smith is working with the PDA sends identification of the smith. Thus, the process control system has information enough to determine which kind of access the smith needs to the component. The process control system returns a list of the parameters the smith can access, in the case of the winch, start, stop, reverse and frequency of the motor, and it suggest a binding of the parameters to standard PDA key. Now, the smith can start, stop and reverse the motor through the up/down scroll button on his own PDA. The overall system consistency is maintained by only letting the smith control locally task and location specific parameters.

This application augments the smith in interacting directly with the motor in a gesturelike fashion. In the workoriented terminology introduced above, this means that the PDA is handled through unconscious, automated operations (scrolling up and down).

It transcends the local control versus system consistency problem by letting the user interact directly on the relevant component through his own interaction device, but still letting the process control system inhibit dangerous violations of interdependencies in the system.

The PDA screen shown in figure 2 is designed for a Palm Pilot. However, an implementation with WAP technology, would probably prove even more suitable.

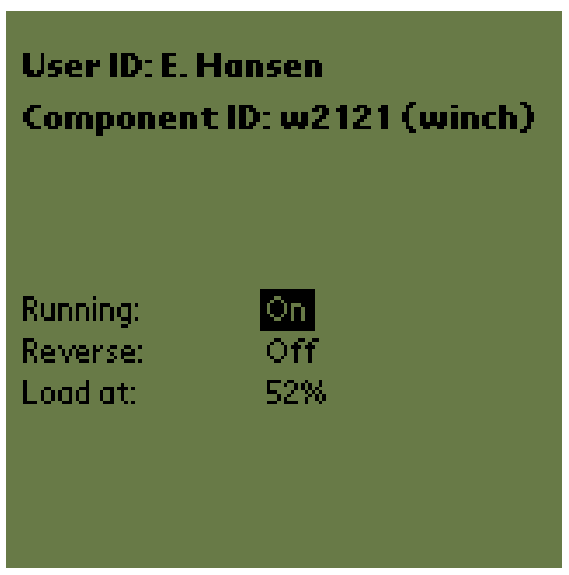

Figure 2: Display from a PDA application based on the augmenting the user strategy

\section{Augmenting the object}

In implementing the principle of augmenting the object in a PDA application supporting work in a wastewater treatment plant we develop two future scenarios. The first scenario is based on the photocopier example above in the sense that it supplies local display and control, the second is based on the concept of augmented paper.

\section{Local display and control}

The first future scenario build on the augmenting the object strategy is derived from the photocopier control display example above. It addresses the lack of local display and control in newer generations of frequency converters

A wastewater treatment plant is equipped with a large number of electrical motors most of which are driven by alternating current. To regulate the speed of such a motor the frequency of the power supply must be regulated by means of a frequency converter.

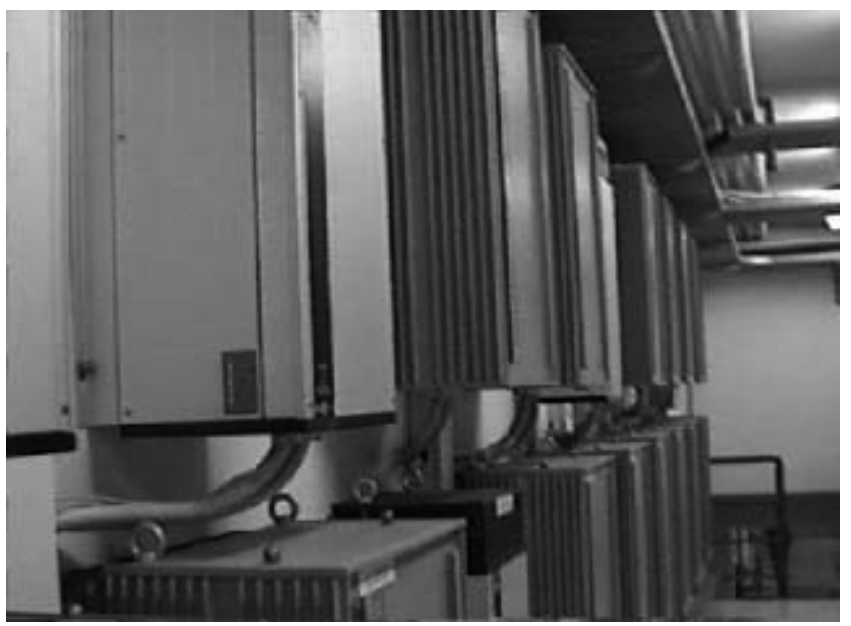

Figure 3: An array of frequency converters

Contrary to the newer models, older frequency converters are often equipped with a small display and controls for operating it directly. The lack of local control and display in the newer models is not a problem; in most situations, it is controlled from a process control system. However, in the occasional situations of breakdown, calibration, maintenance, etc., local control of the unit is needed.

This occasional access to direct operation of the frequency converter can be obtained with a PDA based application augmenting the object, in this case the frequency converter (see figure 4).

The wastewater worker or the external specialist who needs local control of the frequency converter reads the bar code on the unit and attach the PDA and cell phone to the housing. Now, the PDA calls up the process control system through the cell telephone modem, and "negotiates" the amount of control of the frequency converter that can be given locally. Upon initialisation, the controls on the new PDA-based 
local interface consist of the subset of control over the frequency converter it makes sense to have locally, depending on who the person is, which dependencies there are in the actual state of the plant, and the purpose of working on the PDA. Some of these aspects may have been decided beforehand and entered into the process control system centrally.

The frequency converter is augmented with the interface of the PDA that during the situation becomes a part of the component rather than a personal digital assistant. The advantage of this scenario over the direct local control is that dependencies in the plant are not violated by the users' local control. In general, mobile control of process control systems is considered problematic. In this scenario, the dangers are avoided through strong context specificity of control. The cost of the scenario is low because the wastewater treatment plant is extensively wired to the process control system.

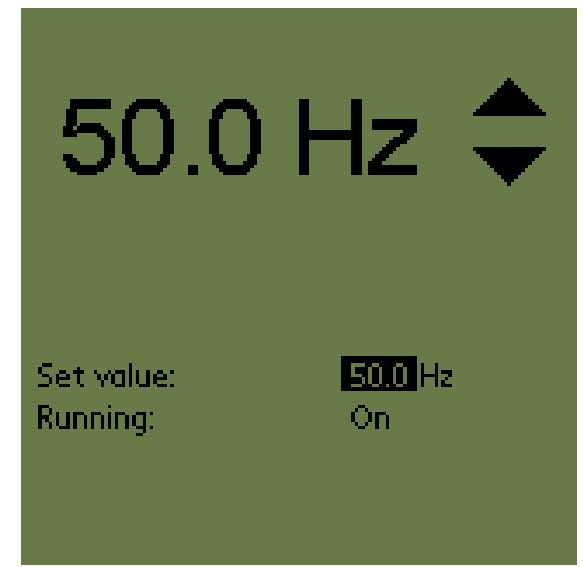

Figure 4: Display from a PDA application based on the local display and control version of the augmenting the object strategy

\section{Augmented paper}

The second future scenario exploring the principle of augmenting the object as a principle in PDA support for wastewater treatment work is based on the concept of augmented paper.

Each day, one of the workers reads the meters related to the production of electricity at the plant. The worker notes the numbers on a protocol sheet, take all the numbers to a note pad on a table performs some calculations, and writes the numbers in a paper record. Finally, he carries some of the numbers on a scrap of paper to the house where the computer is located; there he inputs data to the process control system.

Our study of wastewater treatment work indicated the importance of several hand-written paper records for test results as well as for the gas and electricity production.
These records, however, were also input to the Process control system and therefore the object of rewriting two or three times. This led to discussions of whether the rewriting was a necessary evil caused by the lack of technological support or whether it in fact served other purposes than simply transferring the numbers from one medium to another. To test this hypothesis, we implemented a prototype for an augmented paper record based on the CrossPad technology. The CrossPad was trained to read and recognise the hand-written record and propagate/transfer it the computer system. We set up a scenario where the CrossPad served as a mobile link between the meters and the Process control system, rendering the rewriting obsolete but retaining the paper record. When we presented this solution to workers and managers at the wastewater treatment plant, it was refused. To them, there was no point in hanging on to the paper records if it was possible to solve the authentication issue in another way. Furthermore, they did not believe that rewriting numbers guaranteed that workers actually think about the numbers.

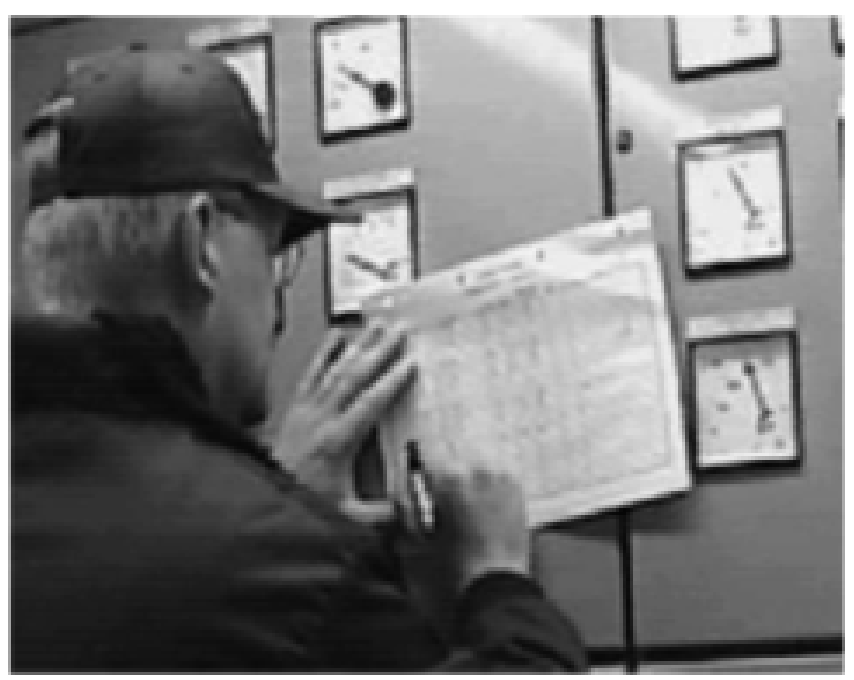

Figure 5: Wastewater operator reading the gas meters

The radical application, based on the augmenting the object strategy, of support for reading the electricity production related meters eliminates the former object and replaces it with the PDA. In the meter-room, the worker reads the bar code on the desk for the PDA to invoke the electricity and gas production record. As he reads the meters, he writes the reading on the number-pad on the PDA screen. Upon reading the meters, the PDA calculate the results and present the worker with the results together with the results from the previous day. The worker leaves the meter-room and goes to the central control room where he "beams" the results into the process control system by way of the infra red port build into the PDA. This application could as well be classified as augmenting the user. 
The PDA would ideally have a number keypad, but for the sake of consistency across examples, we keep with the standard PDA (see figure 6). Again, a WAP based approach is an elegant alternative.

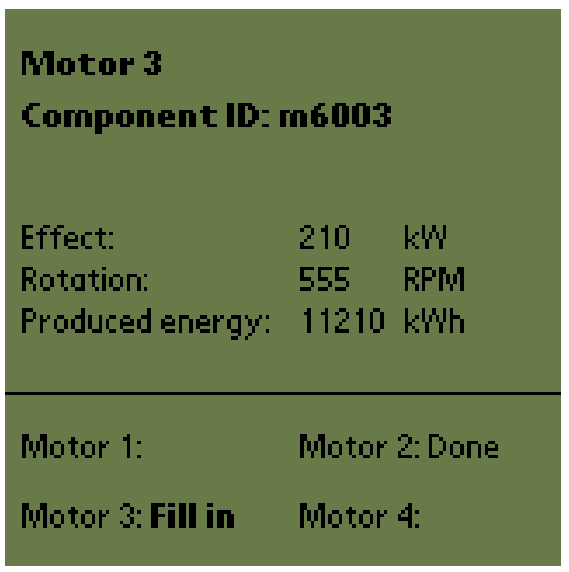

Figure 6: Display from a PDA application based on the local augmented paper version of the augmenting the object strategy

\section{Augmenting the environment}

In implementing the principle of augmenting the environment in a PDA supporting work in a wastewater treatment plant, we take the simple approach of the assembly station, and use it as the basis for the design of an automatic, context sensitive on-line manual.

A wastewater treatment plant is a complex technical system. Becoming familiar with the technical system is not done overnight but is a yearlong learning process. The scope of the task has been very aptly put by one of the unskilled workers at the studied wastewater treatment plant:

"I have been working on this plant for four years and am starting to 'know' the plant, but I think it will take another two years before I know it well enough - where all the machines are, etc. For example, we have pipes running through this building and they are normally working fine so you can basically work here for years without knowing there's a (throttle) valve up there [pointing]" (..) You never find out before something happens (..) We work with 50.000 components - you don't learn about them on your first day".

One of the responsibilities of this specific worker is to mind an array of motors producing electricity from gas produced from sludge in the plant. Maintenance of the gas motors is divided between workers at the plant, the municipal blacksmith department, and technicians from the manufacturer. Minor repair and maintenance tasks performed by one of the unskilled workers are coordinated with major repair, inspection and maintenance, in order to reduce down time. One of the frequent tasks is the cleaning and adjustment of spark plugs in the motor.

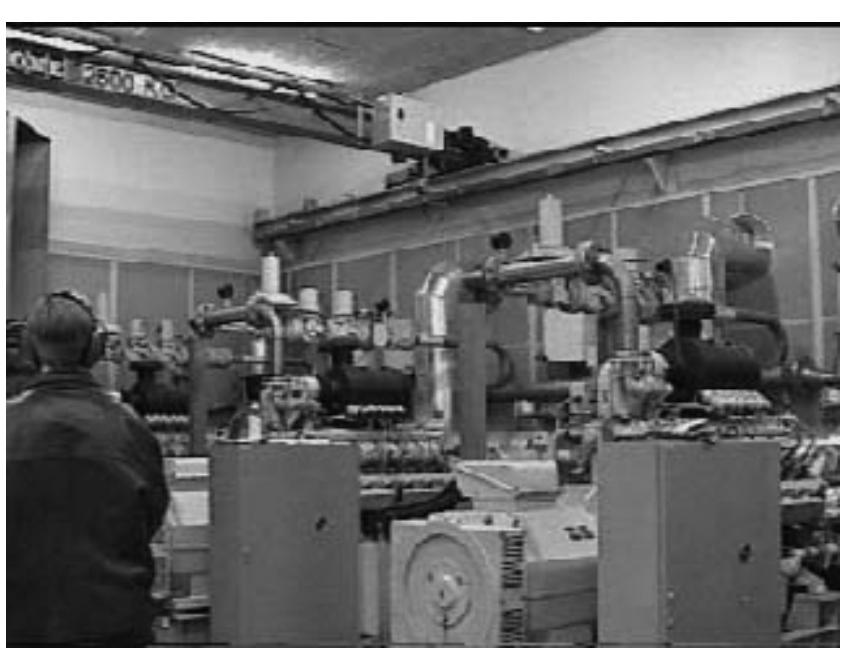

Figure 7: The gas motors

The principle of augmenting the environment through the PDA may be implemented to aid this assignment in the following manner. The wastewater treatment worker enters the area with the gas motors and starts the procedure by turning off the relevant motor manually. He then scans the bar code on the motor with his PDA, connects to the process control system through the mobile phone attached to it and checks on it that the shut down has been acknowledged there. He places the PDA on a shelf next to the motor so the display is visible from his current position. The PDA displays a simple blueprint-type drawing of the motor, highlighting the front panel, and a short textual description of how to remove it (see figure 8). After having removed the front panel he runs its bar code past the scanner on the PDA; the display changes to show the next step of the disassembly procedure. In this manner, he works his way into the motor to the spark plugs. The PDA presents instructions in which order to remove and replace the spark plugs, triggered by scanning the individual units. He then acknowledges on the PDA that no more motor components will need replacement, and is now guided through the procedure of reassembling it, scanning the components in reverse order. For users experienced with a specific type of motor and task, the online manual serves only as a peripheral guide with the purpose of making sure the relevant motor components are removed and (in particular) re-inserted. For novice users, the online manual may serve as a component specific, context dependent learning guide.

This future scenario illustrates how we may support the vast learning task involved in maintaining the multitude of components in this wired wilderness through externalising the relevant information through the PDA. 
A far more elegant solution, but far from realistic today, for this application would be to embed a camera with effective pattern recognition features to seamlessly do what the worker in the example has to do by hand and bar code reader.

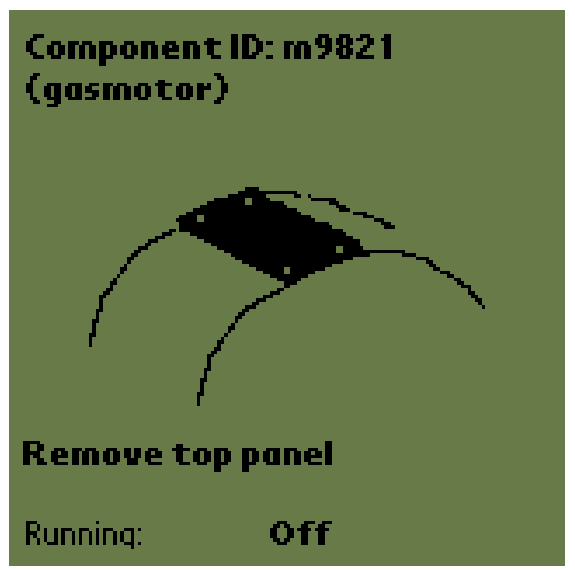

Figure 8: Display from a PDA application based on the augmenting the environment strategy

\section{DISCUSSION}

We have presented future scenarios for wastewater treatment work with PDA applications developed by using the technical classification of augmented reality interfaces as a thinking tool. The applications in these scenarios are designed for a standard PDA extended with a bar code reader and a modem for a cellular telephone. Thus, we have been emphasising solutions that can be implemented with limited resources today. The use of bar codes is clumsy, but has the advantage of being cheap, and making the choice of context totally explicit. In this respect, our contribution is in radical contrast to achievements like the Cy-Phone, which is an all-purpose personal tool based on Pico Cells [13]. Our future scenarios address some of the specifics of wastewater treatment work: the distributedness, the number of different component the workers have to deal with, the wired wilderness enabling most of the designs. In a setting with a lower degree of interdependency and "wiredness", PDA support might be different. However, the principle of designing PDA applications as augmentation of the user, physical objects or the environment is likely to be applicable in a broader context.

The applications in the four future scenarios have been developed into horizontal prototypes, i.e. screen layouts with no underlying functionality. In general horizontal prototypes and mock-ups are suitable means of testing design ideas with users. However, in this particular case, we believe that some amount of functionality and integration with the process control system is needed investigate into the ideas beyond the level waving our arms. This is an obvious problem because outside interaction with a control system can have dangerous effects, and because it requires access to the control system software.

Using the concept of augmentation and the classification of augmented reality interfaces as a thinking tool gave us several advantages. Using the augmented reality principles as thinking tools sparked our imagination and at the some time steered us clear of pure science fiction.

Initially, the concept "augmented reality" seemed to be impossible to apply in PDA design; it seemed that a PDA application would always end up being based on the "augmenting the user" class of interfaces. This apparent impossibility turned out to be an important source of creativity. This technique is generally applicable. That is, when established design principles impose tunnel vision on designers, proceed by selecting technical concepts that obviously not fit the design problem, and then structuring the new solutions according to the misfit concepts.

We saw that the classification of augmented reality interfaces seemed to blur when applied to PDA design. However, taking the second scenario as an example, even if the PDA based record looks like an augmentation of the user the design is still based on automating and augmenting the paper based record. This seeming confusion is due to augmented reality being a pragmatic technical classification of a class of interfaces technologies.

Focussing on interaction with physical objects in the work setting provided us with specific boundaries to work within and thus became an effective guide in deciding what to include in each of the PDA applications. Thus, the concepts of augmented reality worked as a tool for handling the context problem. Most of the information and control made accessible with the suggested PDA applications is present in the existing process control system. The strong focus on interaction with physical objects around the plant, however, helped in specifying actual physical and situational context for the applications in the future scenarios.

The three different strategies of augmenting the user the object and the environment enabled us to transcend the image of interaction with a PDA as the users introvert fiddling with his little pen on his personal four square inch pad. Through our future scenarios, a broader range of physical postures in interaction with a PDA emerged: hand waving, writing, walking around, etc. The original formulation of augmented reality was primarily a concept of interface technology, the way we have used it in this paper turns it into an interaction style concept. Thus, augmented reality may be abstracted into interaction concept independent of the concrete technical substrate of implementation.

As stated in the introduction, direct manipulation as a general user interface principle falls short in relation to mobile devices. In particular the lack of screen space 
inhibits detailed representation of the object of work at the screen. Basing PDA design on concepts of augmented reality interfaces, forced us to think beyond direct manipulation; it simply does not make sense to represent the object of work naturalistically on the screen if it is physically present in the situation of work. However, as illustrated in our future scenarios, principles of direct manipulation interfaces, e.g. directness and transparency, apply nicely for mobile devices just as well as for fullfledged augmented reality systems.

\section{ACKNOWLEDGMENTS}

We thank the people at the wastewater treatment plant for their time and effort. Susanne Bødker, Wendy Mackay, Astrid Søndergaard and Christian Yndigegn have all been a great inspiration in the wastewater project. Christian Yndigegn programmed the augmented paper prototype for the CrossPad. We acknowledge the effort of the people at Danfoss and at Malmö University College. The Danish Basic Research Foundation, Centre for Human-Machine Interaction and The Danish National Centre for IT-Research, project no. 23 (Usability Work in the Danish Industry) have supported our work on the wastewater project.

\section{REFERENCES}

1. Bærentsen, K. (1989). Menneske og maskine [Man and Machine]. In Hedegaard, Hansen \& Thyssen (eds.). Et virksomt liv [An active life]. Aarhus: Aarhus University Press, pp. 142-187.

2. Bertelsen, O. W. (1996). The Festival Checklist: design as the transformation of artefacts. In Blomberg, J., Kensing, F. \& Dykstra-Erickson (eds.). PDC '96, Proceedings of the Participatory Design Conference, Palo Alto: Computer Professionals for Social Responsibility, pp. 93-101.

3. Bertelsen, O.W. \& C. Nielsen (1999). Dynamics in Wastewater Treatment: A Framework for Understanding Formal Constructs in Complex Technical Settings. In Bødker, S., M. Kyng \& Kjeld Schmidt (eds.). ECSCW '99, Proceedings of the Sixth European Conference on Computer Supported Cooperative Work, 12-16 September 1999, Copenhagen, Denmark. Dordrecht: Kluwer. pp. 277-290.

4. Bødker, S. (1991). Through the Interface: a human activity approach to user interface design. Hillsdale: Lawrence Erlbaum Associates.

5. Dix, A., K. Chervest, N. Davies and T. Rodden (1998). Exploiting context in HCI design for mobile systems. In Johnson, C. (ed.) Proceedings of First Workshop on
Human Computer Interaction for Mobile Devices. Department of Computing Science, University of Glasgow.

6. Engeström, Y. (1987). Learning by expanding: an activity-theoretical approach to developmental research. Helsinki: Orienta-Konsultit Oy.

7. Johnson, P (1999). Usability and Mobility; Interactions on the move. In Proceedings of the Second Workshop on Human Computer Interaction with Mobile Devices, 31 August 1999, Edinburgh, Scotland.

8. Kristoffersen, S. and F. Ljungberg (1998). "Representing modalities in mobile computing," In B. Urban, T. Kirste and R. Ide (eds.) Proceedings of Interactive applications of mobile computing. Fraunhofer: Institute for Computer Graphics.

9. Kristoffersen, S. \& Ljungberg F. (1999). "Making Place" to Make IT Work: Empirical Explorations of HCI for Mobile CSCW. In Proceedings of International Conference on Supporting Group Work (GROUP'99), ACM Press.

10. Mackay, W. (1998). Augmenting Reality: Linking real and virtual worlds A new paradigm for interacting with computers. In Proceedings of AVI'98, ACM Conference on Advanced Visual Interfaces, New York: ACM Press.

11. Madsen, K.H. (1994). A guide to Metaphorical Design. The Communications of the ACM, pp. 57-62.

12. Olsen, D. Interacting in chaos (1999). In Interactions Vol. 6, No. 6 (Nov. 1999), pp. 42-54.

13. Pulli, P., T. Pyssysalo, K. Kuutti, J. Similä, J-P Metsävainio and O. Komulainen (1998) CyPhoneFuture Personal Telecooperation Device. In Proceedings of the XV IFIP World Computer Congress, Vienna, Austria and Budapest, Hungary, August 31 - September 4, 1998, pp. 529-540.

14. Winograd, T. \& Flores, F. (1986). Understanding Computers and Cognition -- a new foundation for design. Norwood, N.J: Ablex.

15. Yamazaki, K., A. Yamazaki, h. Kuzuoka, S. Oyama, h. Kato, H. Suzuki, H. Miki (1999). GestureLaser and GestureLaser Car: Development of an embodied space to support remote instruction. In Bødker, S., M. Kyng \& Kjeld Schmidt (eds.). ECSCW '99, Proceedings of the Sixth European Conference on Computer Supported Cooperative Work, 12-16 September 1999, Copenhagen, Denmark. Dordrecht: Kluwer, pp 239-258. 